\title{
Measuring real life motor activity in depression
}

\author{
Oury Chocron ${ }^{1}$, Selma Aybek ${ }^{2,3}$, Anisoara Paraschiv-Ionescu ${ }^{4}$, Kamiar Aminian $^{4}$, François Vingerhoets $^{2}$ and Alexandre Berney $^{1 *}$ \\ ${ }^{1}$ Psychiatric Liaison Service, Lausanne University Hospital, Lausanne, Switzerland \\ ${ }^{2}$ Clinical Neurosciences Department, Lausanne University Hospital, Lausanne, Switzerland \\ ${ }^{3}$ Neurology University Clinic, University Hospital Inselspital, Bern, Switzerland \\ ${ }^{4}$ Laboratory of Movements Analysis, Federal Institute of Technology EPFL, Lausanne, Switzerland
}

\begin{abstract}
Introduction: Motor alterations associated to Major Depression remain to date poorly characterized. Our group developed a new method allowing to objectively measure several dimensions of motor activity in a "real life" setting. The aim of this study was to measure spontaneous motor activity in depressed patients compared with healthy subjects.
\end{abstract}

Methods: Ten depressed patients $(46 \pm 20$ years) and ten age- and gender-matched healthy controls $(48 \pm 21$ years $)$ underwent a 6 -h ambulatory monitoring of spontaneous motor recording with a validated wireless device.

Results: Depressed subjects showed objective motor alterations compared to healthy controls. Speed and quantity of hand movements were both reduced, and a positive correlation was found between severity of depression and reduction in movements' quantity. Depressed subjects also showed a slowing of gait, as illustrated by a reduced cadence, longer double limb support and swing time. Depressed subjects spent more time in a lying position, as opposed to an active position such as walking or standing and less time outdoors.

Discussion: These results demonstrate that motor alterations in depression can be objectively monitored with a wireless device. This novel way of studying motor changes might be applicable in the future in the identification of endophenotypes of depression and potential predictors of treatment response.

\section{Introduction}

Psychomotor retardation is one of the features of Major Depression and is part of the diagnostic criteria in most classification systems. Although motor alteration has clinical and possibly prognostic implication $[1,2]$, this dimension remains to date poorly characterized [3].

Specific scales have been validated for clinical assessment of psychomotor retardation in depression [4,5], but have the disadvantage of being observer dependent. Objective measurements were developed [6], but most of them need an in-laboratory setting and only capture data in a testing condition. However, depressive symptoms are known to present diurnal fluctuations [7]. To overcome this problem, actigraphic measurements have been used to monitor motor activity in a "real life" setting [8]. Although actigraphic measurements provide data on the number of movements per epoch, they do not measure speed of movements. Altogether, a recent review [3] concluded that data on the status of psychomotor retardation in depression are still fragmentary and confirm the need of further quantitative and qualitative investigations. More advanced methods for objective motor assessment may allow improving our understanding of motor alterations in depression.

Our group developed a wearable monitoring system, validated in several clinical samples [9-12]. It allows to objectively measure several dimensions of motor activity in a "real life" setting. Using this method in a previous study [13], we reported an alteration of the temporal organization of motor activity in patients with major depression. In this paper, we report additional motor activity parameters measured in this sample of depressed patients, including gait, body posture and proportion of outdoor activity.
The aim of this study was to assess "real life" motor activity in depression using the monitoring system and algorithm devised to quantify several parameters of motor activity throughout the day. Our hypothesis is that depressed patients will present altered motor parameters (hand, gait, body position, outdoor activity) as well as decreased motor activity compared to healthy controls.

\section{Methods}

The sample and the procedure are the same as those described in a previous publication [13]. We present here the results of additional analyses of motor activity measurements.

\section{Subjects}

Ten consecutive patients aged between 18 and 65 years, who were diagnosed with current major depressive episode (according to DSM-IV-TR criteria) and scored $>21$ on the Hamilton Rating Scale for Depression (HRSD), were recruited from the outpatient Psychiatry clinic at Lausanne University hospital. Patients on antidepressant, anxiolytic, hypnotic or antipsychotic medication in the last 3 months were excluded, in order to prevent pharmacological influence on motor activity. Patients suffering from any other psychiatric disorder (most notably bipolar affective disorder) and/or from another condition that could possibly interfere with motor activity (neurological disorders,

${ }^{\star}$ Correspondence to: Alexandre Berney, Associate Professor, Department of psychiatry, Lausanne University Hospital, Switzerland, E-mail: Alexandre. Berney@chuv.ch

Received: July 22, 2020; Accepted: August 13, 2020; Published: August 15, 2020 
orthopedic diseases, general condition or substance abuse) were also excluded. Age and gender matched healthy controls were recruited from the general population.

Signed informed consent was obtained for each subject and the study protocol was approved by the Lausanne University Hospital ethics committee.

\section{Procedure}

\section{Mood assessment}

Diagnosis of depression was assessed by a psychiatrist according to DSM-IV criteria using the MINI interview and mood was assessed in all subjects using the Hamilton Rating Scale for Depression (HRSD 17 items) and the Beck Depression Inventory (BDI 21 items).

\section{Motor assessment}

Measurement protocol: Motor activity was assessed using five synchronized wearable devices $[11,13]$, each including inertial sensors (3-axis accelerometer and 3-axis gyroscope), rechargeable battery and memory for data recording at $200 \mathrm{~Hz}$ sampling rate. Devices were lightweight $(50 \mathrm{~g})$ and were attached to the body with medical patches without hindering the subject, so that daily activities were performed normally. The five devices were placed on the body as follows: one on each wrist to record hand activity, one on each ankle to record walking parameters, and one on the trunk to record posture parameters. Subjects were instructed to carry on with their usual daily activities. We specifically did not impose or forbid any activity, as the aim of the study was to record spontaneous activity and we wanted the subjects to move freely. Both depressed and healthy subjects were recorded during a non-working day. The time of spontaneous recording was between 10 am and $4 \mathrm{pm}$ for every subject.

\section{Motor activity analysis}

Hands motor activity was quantified based on analysis of hand angular velocities and included the following steps: conditioning of gyroscope signal (drift cancellation), identification of movement periods and calculation of parameters related to identified movements. Mobility represented the average velocity of hands and was estimated from the norm of angular velocity signals recorded by the gyroscope (square root of the sum of square of 3-axis signals). Activity was defined as the percentage of time the hand was moving and the range of rotation represented the rotation angles of hands during the periods of movement. These parameters (mobility, activity and range of rotation) were calculated on epochs of 1-min length. Gait parameters were estimated based on angular velocity signals recorded by the gyroscopes on the ankles. Spatio-temporal parameters were extracted based on estimation of heel strike and toe-off times. The timing of these events allowed the measurement of temporal gait parameters such as: cadence, swing, stance and double support durations [9]. The algorithm was validated against camera-based system (ELITE) using a database of recorded signals from normal and pathological gait. Stride to stride variability of gait parameters was estimated using the coefficient of variation (CV). Body position (i.e. lying, standing and walking) was estimated from acceleration and angular velocity signals recorded from trunk and ankles devices [11]. Using this algorithm, the sitting and standing periods were detected using the kinematic features of trunk movements during transitions between sitting and standing postures, and a statistical classifier. Lying periods were calculated from the level of trunk acceleration signals. Walking episodes were detected from the pattern of shank angular velocity signals. Outdoor activity was estimated from successive long walking episodes (superior to 30 seconds) recorded during the monitoring time. The hypothesis was that continuous walking for more than $30 \mathrm{sec}$ is unlikely to be performed in home/indoor environments. The periods of monitoring including temporal clusters of long walking episodes (i.e. episodes that succeed at short intervals) were assigned as outdoor activity.

\section{Statistical analysis}

Differences between groups (depressed vs. healthy controls) were assessed for demographic data (using Student's t-test) and motor activity parameters (non-parametric Mann Whitney test). These parameters were estimated for each subject, and their mean and standard deviation were calculated within each group. Relationships between depression scores and motor activity parameters were assessed with Spearman's rank correlation coefficient. A value of $\mathrm{P}<0.05$ was considered significant.

\section{Results}

\section{Description of the sample}

Depressed patients were $46.6 \pm 20.0$ years old and healthy controls $48.3 \pm 21.6(p=0.8)$. In both groups, 6 female and 4 men were recruited. Depressed subjects scored $30.8 \pm 4.1$ on HRSD (healthy controls: $3.0 \pm 2.1 ; p<0.01^{\star}$ ) and $37.5 \pm 11.4$ on BDI (healthy controls: $2.7 \pm 2.9$; $\left.p<0.01^{\star}\right)$, corresponding to severe major depression.

\section{Hand motor parameters}

Hand mobility (speed in deg/s) and hand activity (\% of movements per 1 min-epoch) were significantly reduced in both hands in depressed subjects compared to healthy controls. Healthy subjects scored $57.54 \pm 7.50 \mathrm{deg} / \mathrm{s}$ and $5911 \mathrm{deg} / \mathrm{s}$ for right hand and left hand mobility respectively, while depressed patients scored $42.3 \pm 13.5 \mathrm{deg} / \mathrm{s}$ $\left(p=0.044^{\star}\right)$ and $39.7 \pm 13.5 \mathrm{deg} / \mathrm{s}\left(p=0.020^{\star}\right)$. Healthy subjects scored $0.62 \pm 0.11 \%$ and $0.60 \pm 0.10 \%$ for right hand and left-hand activity respectively, while depressed patients scored $0.47 \pm 0.24 \%\left(p=0.030^{*}\right)$ and $0.42 \pm 0.21 \%\left(p=0.018^{*}\right)$. No difference was observed in range of rotation in both hands between the two groups. There was a significant negative correlation $\left(r=-0.48, p=0.03^{*}\right)$ between Hamilton depression score and level of hand activity: the more depressed the subject, the lower the hand activity (Figure 1).

\section{Gait parameters}

A significant reduction in gait speed and cadence was observed in depressed subjects (Table 1) compared to healthy controls with depressed subjects scoring $1.1 \pm 0.1 \mathrm{~m} / \mathrm{s}$ in speed and $51 \pm 2 \mathrm{steps} / \mathrm{min}$ in cadence, while healthy controls scored $1.4 \pm 0.09 \mathrm{~m} / \mathrm{s}\left(p=0.001^{*}\right)$ and $60 \pm 3$ steps $/ \mathrm{min}\left(p=0.001^{\star}\right)$ respectively. Depressed subjects displayed a significantly longer gait cycle time $(1.16 \pm 0.09 \mathrm{~s}$ compared to $1 \pm 0.05$ $\left.\mathrm{s} ; p=0.000 .6^{*}\right)$, longer double support time $(26 \pm 6.1 \mathrm{~s}$ compared to $\left.21 \pm 2 \mathrm{~s} ; p=0.02^{*}\right)$, and longer swing time $(39 \pm 1.2 \mathrm{~s}$ compared to $36 \pm 2$ $s ; p=0.01^{\star}$ ) than healthy controls (Table 1$)$. Depressed patients also had a significantly higher variability for both gait cycle time $(4 \pm 1.79 \%$ compared to $\left.2.1 \pm 0.5 \% ; p=0.01^{*}\right)$ and swing time $(5.2 \pm 2.8 \%$ compared to $3.1 \pm 2 \% ; p=0.001^{\star}$ ) than healthy controls (Table 1 ).

\section{Body position and outdoor activity}

Depressed patients spent $23 \pm 18 \%$ of the monitoring duration lying down while healthy subjects spent only $8 \pm 15 \%\left(p=0.01^{\star}\right)$. However, there was no significant $(p=0.1)$ difference in time walking or standing between the two groups (Table 1). Depressed subjects spent a significant greater amount of time indoors than controls and less time outdoors 

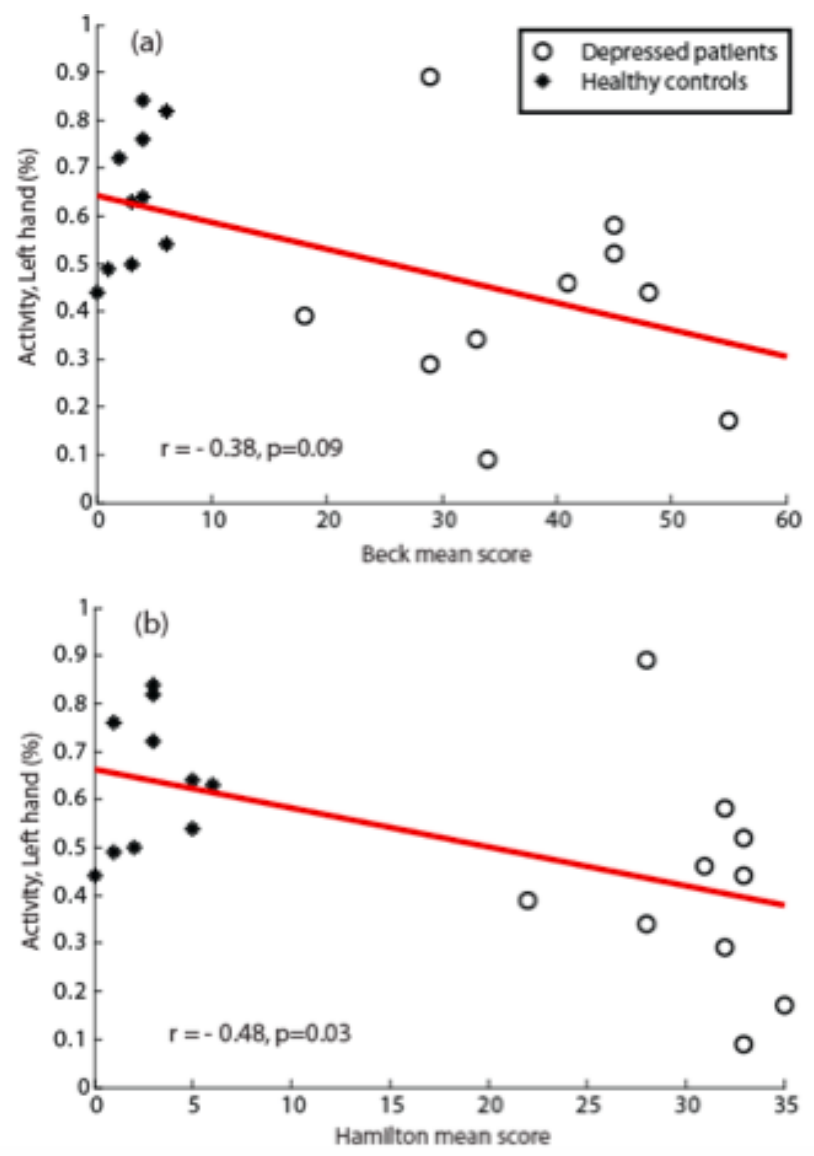

Figure 1. Hand activity and mood scores. Relationships between hand motor activity and mood rating scores for depressed and healthy subjects. Significant correlations were obtained between hand activity and Hamilton mean score $\left(r=-0.48, p=0.03^{*}\right)$

Table 1. Gait parameters, body position and outdoor activity (Two-sided Mann Whitney test) Results are expressed in mean \pm standard deviation

\begin{tabular}{|c|c|c|c|}
\hline & $\begin{array}{l}\text { Depressed patients } \\
\qquad(\mathrm{n}=10)\end{array}$ & $\begin{array}{l}\text { Healthy controls } \\
\qquad(\mathrm{N}=10)\end{array}$ & P-value \\
\hline \multicolumn{4}{|l|}{ Gait parameters } \\
\hline Speed $(\mathrm{m} / \mathrm{s})$ & $1.1 \pm 0.1$ & $1.4 \pm 0.09$ & $0.001 *$ \\
\hline Cadence (steps/min) & $51 \pm 2$ & $60 \pm 3$ & $0.001^{*}$ \\
\hline Gait cycle time (s) & $1.16 \pm 0.09$ & $1 \pm 0.05$ & 0.0006 \\
\hline Double support (s) & $26 \pm 6.1$ & $21 \pm 2$ & 0.02 \\
\hline Swing time (s) & $39 \pm 1.2$ & $36 \pm 2$ & $0.01 *$ \\
\hline \multicolumn{4}{|c|}{ Gait variability parameters } \\
\hline $\begin{array}{c}\text { CV Gait cycle time } \\
(\%)\end{array}$ & $4 \pm 1.79$ & $2.1 \pm 0.5$ & $0.01 *$ \\
\hline CV Swing time (\%) & $5.2 \pm 2.8$ & $3.1 \pm 2$ & $0.001 *$ \\
\hline \multicolumn{4}{|c|}{ Physical activity parameters in \% of monitoring duration } \\
\hline Lying & $23 \pm 18$ & $8 \pm 15$ & $0.01 *$ \\
\hline Standing \& walking & $37 \pm 15$ & $40 \pm 12$ & 0.1 \\
\hline Outdoor activity & $8.3 \pm 5.1$ & $14.4 \pm 5$ & $0.03 *$ \\
\hline
\end{tabular}

with an outdoor activity percentage of $8.3 \pm 5.1$ compared to healthy controls at $14.4 \pm 5\left(p=0.03^{\star}\right)$ (Table 1$)$.

\section{Discussion}

This cross-sectional controlled study demonstrated that when measured objectively in a spontaneous real life setting depressed subjects have significant motor alterations compared to healthy controls. Speed and quantity of hand movements were both reduced, and a positive correlation was found between severity of depression and reduction in movements' quantity. Depressed subjects also showed a slowing of gait, as illustrated by a reduced cadence, longer double limb support and swing time. Depressed subjects spent more time in a lying position, as opposed to an active position such as walking or standing and less time outdoors. It is important to note that all subjects tolerated the device and no subjects dropped out of the study. Altogether, our method proved to be easy to use and patients were not hindered by the recording system and could carry on with their usual activities.

These results obtained for the first time in "real life" setting recording simultaneously several measures of motor activity, each one composed of multiple parameters confirms previous results obtained in in-laboratory studies [14,15] and actigraphy studies [8]. This allows a more precise description of motor alterations in depression allowing ultimately a better understanding of this phenomenon. The significant correlation between reduction in hand movements' quantity and severity of depression as measured by Hamilton Rating Scale for Depression (HRSD) reflects the fact that motor alterations seem to be state dependent.

Decreased activity in depression is usually understood as the result of a lack of interest and motivation influenced by the affective dimension. However, results presented here as well as in our previous publication reveal qualitative motor alterations as well as alterations in temporal organization pattern [13], more complex than a simple overall decrease in activity. In terms of neurobiological correlates of motor alteration in major depression, a recent review focusing on neuroimaging findings conclude that both structural and functional alterations are present [16]: White matter hyperintensities as well as thinning of right presupplementary motor cortex (pre-SMA) were found to correlate with psychomotor alterations. Moreover, evidence suggests decreased metabolism in Dorso-lateral Prefrontal Cortex (DLPFC) and increased Anterior Cingulate Cortex (ACC) metabolism, as well as lowered dopaminergic tone and altered metabolism in striatal regions $[17,18]$. Furthermore, recent studies suggested that decreased psychomotor speed in major depressive disorder could be linked to the inflammation of basal ganglia [19] and showed that inflammatory cytokines affect the basal ganglia and dopamine neurotransmission to mediate depressive symptoms related to motivation and motor activity [20]. Altogether these alterations could result in the complex motor changes observed in depressed patients.

Our study has several limitations. First, our sample size is small; our results are however robust with significant group differences and this could serve to run power analyses to conduct further bigger studies. Second, as we chose to give minimal instructions to the participants - they just had to live a "normal day" during recording - one cannot exclude that being recorded has influenced some of the participants in their daily program. Measuring several days in each participant in the future might minimize such influence. Furthermore, in order to limit a working/non-working bias, as depressed subjects were on sick leave, we recorded all subjects on a non-working day.

\section{Conclusion}

Although the results are promising, more extended studies are necessary to better define motor changes in depression. Such data may allow determining phenotypes or specific clinical subtypes of depression [21], which may be key to a better understanding of the physiopathology of this complex and heterogeneous disorder. Furthermore, such measurements could advance treatment selection and help predict the 
outcome of depressed patients [1,2]. In future studies, a longitudinal design could provide valuable information on the temporal evolution of motor changes during the course of treatment in depressed subjects.

\section{References}

1. Mccall WV (2015) A rest-activity biomarker to predict response to SSRIs in major depressive disorder. J Psychiatr Res 64: 19-22. [Crossref]

2. Michael P Caligiuri, Valentina Gentili, Sonja Eberson, John Kelsoe, Mark Rapaport, et al. (2003) A quantitative neuromotor predictor of antidepressant non-response in patients with major depression. J Affect Disord 77: 135-141.

3. Bennabi D, Pierre Vandel, Charalambos Papaxanthis, Thierry Pozzo, Emmanuel Haffen (2013) Psychomotor retardation in depression: a systematic review of diagnostic, pathophysiologic, and therapeutic implications. Biomed Res Int 2013: 158746.

4. Parker G, D Hadzi-Pavlovic, K Wilhelm, I Hickie, H Brodaty (1994) Defining melancholia: properties of a refined sign-based measure. Br J Psychiatry 164: 316-326.

5. Widlocher DJ (1983) Psychomotor retardation: clinical, theoretical, and psychometric aspects. Psychiatr Clin North Am 6: 27-40. [Crossref]

6. Sabbe B, W Hulstijn, J van Hoof, H G Tuynman-Qua, F Zitman (1999) Retardation in depression: assessment by means of simple motor tasks. J Affect Disord 55: 39-44.

7. Wirz-justice A (2006) Biological rhythm disturbances in mood disorders. Int Clin Psychopharmacol 21 Suppl 1 : S11-S15.

8. Raoux N, O Benoit, N Dantchev, P Denise, B Franc, et al. (1994) Circadian pattern of motor activity in major depressed patients undergoing antidepressant therapy: relationship between actigraphic measures and clinical course. Psychiatry Res 52: 8598.

9. Salarian A, Heike Russmann, François J G Vingerhoets, Catherine Dehollain, Yves Blanc, et al. (2004) Gait assessment in Parkinson's disease: toward an ambulatory system for long-term monitoring. IEEE Trans Biomed Eng 51: 1434-1443. [Crossref]

10. Salarian A, Heike Russmann, François J G Vingerhoets, Pierre R Burkhard, Kamiar Aminian (2007) Ambulatory monitoring of physical activities in patients with Parkinson's disease. IEEE Trans Biomed Eng 54: 2296-2299.
11. Salarian A, Heike Russmann, Christian Wider, Pierre R Burkhard, Françios J G Vingerhoets, et al. (2007b) Quantification of tremor and bradykinesia in Parkinson's disease using a novel ambulatory monitoring system. IEEE Trans Biomed Eng 54: 313-322.

12. Aminian K, B Najafi, C Büla, P-F Leyvraz, Ph Robert (2002) Spatio-temporal parameters of gait measured by an ambulatory system using miniature gyroscopes. $J$ Biomech 35: 689-699.

13. Aybek S, Ionescu A, Berney A, Chocron O, Aminian K, et al. (2012) Fractal temporal organisation of motricity is altered in major depression. Psychiatry Res 200: 288-293.

14. Radovanovic S, Milica Jovičić, Nadja P Marić, Vladimir Kostić (2014) Gait characteristics in patients with major depression performing cognitive and motor tasks while walking. Psychiatry Res 217 : 39-46. [Crossref]

15. Lemke MR, T Wendorff, B Mieth, K Buhl, M Linnemann (2000) Spatiotemporal gai patterns during over ground locomotion in major depression compared with healthy controls. J Psychiatr Res 34: 277-283.

16. Liberg B, Christoffer Rahm (2015) The functional anatomy of psychomotor disturbances in major depressive disorder. Front Psychiatry 10: 6-34.

17. Meyer JH, Heather E McNeely, Sandra Sagrati, Anahita Boovariwala, Krystle Martin (2006) Elevated putamen D (2) receptor binding potential in major depression with motor retardation: an $[11 \mathrm{C}]$ raclopride positron emission tomography study. $\mathrm{Am} \mathrm{J}$ Psychiatry 163: 1594-1602

18. Walther S, O Höfle, A Federspiel, H Horn, S Hügli, et al. (2012) Neural correlates of disbalanced motor control in major depression. J Affect Disord 136: 124-133.

19. Goldsmith DR, Ebrahim Haroon, Bobbi J. Woolwine, Moon Y. Jung, Evanthia C. Wommack, et al. (2016) Inflammatory markers are associated with decreased psychomotor speed in patients with major depressive disorder. Brain Behav Immun 56: 281-288.

20. Felger JC (2016) The Role of Dopamine in Inflammation-Associated Depression: Mechanisms and Therapeutic Implications. Curr Top Behav Neurosci.

21. Faurholt-jepsen M, Søren Brage, Maj Vinberg, Ellen Margrethe Christensen, Ulla Knorr, et al. (2012) Differences in psychomotor activity in patients suffering from unipolar and bipolar affective disorder in the remitted or mild/moderate depressive state. J Affect Disord 141: 457-463. [Crossref]

Copyright: (C2020 Chocron O. This is an open-access article distributed under the terms of the Creative Commons Attribution License, which permits unrestricted use, distribution, and reproduction in any medium, provided the original author and source are credited. 